\title{
Nutrition Therapy in Critically Ill Overweight Elderly Patient with Heart Failure, Myocardial Infarction, Pneumonia, and Chronic Kidney Disease
}

\author{
Christina RusLI ${ }^{1}$, Agussalim BuKHARI ${ }^{2}$, Nurpudji A. TASLIM ${ }^{2}$, \\ Suryani As' $\mathrm{AD}^{2}$ and Haerani RASYID ${ }^{2,3}$ \\ ${ }^{1}$ Clinical Nutrition Specialist Program, Faculty of Medicine, Hasanuddin University, Makassar, Indonesia \\ ${ }^{2}$ Department of Nutritional Science, Faculty of Medicine, Hasanuddin University, Makassar, Indonesia \\ ${ }^{3}$ Department of Internal Medicine, Faculty of Medicine, Hasanuddin University, Makassar, Indonesia
}

(Received June 23, 2019)

\begin{abstract}
Summary Overweight or obesity will increase the risk of morbidity and mortality from cardiovascular disease. In older people, the risk is higher, but also paradoxically associated with lower mortality rates. Overweight patients vary in body composition and when it coupled with limited reliable sources to make caloric requirements estimation will make nutrition therapy extremely challenging. This case study reveals the nutrition therapy support in critically ill overweight elderly patient with heart failure, myocardial infarction, pneumonia, and chronic kidney disease. An 80-year old moderate malnourished male patient (body mass index $24.6 \mathrm{~kg} / \mathrm{m}^{2}$ ) with acute lung edema, cardiogenic shock, myocardial infarction, pneumonia, and chronic kidney disease was admitted in the cardiovascular intensive-care unit. The patient was treated with diuretics, vasopressor support, and antibiotics. Oral intake was reduced due to shortness of breath and loss of appetite. The physical examination revealed basal lung rales, wheezing, muscle wasting, edema. Blood tests showed hyperkalemia, leucocytosis, depletion of the immune system, hyperuricemia, hypoalbuminemia, and dyslipidemia. The patient was on stage 5 renal failure (GFR $6.2 \mathrm{~mL} / \mathrm{min}$ ) but refused hemodialysis treatment. Nutritional therapy was given gradually with calorie target $1900 \mathrm{kcal}$ and protein $0.6-1.2 \mathrm{~g} /$ ideal body weight/d using normal foods, oral nutrition supplement, and amino acids parenteral nutrition. After $13 \mathrm{~d}$ of nutritional treatment, the patient was discharged from the hospital with no shortness of breath, adequate nutrition intake, increased renal function (GFR $22.4 \mathrm{~mL} / \mathrm{min}$ ), and improvement of the blood test results (immune status, uric acid, albumin, and lipid profile). Critically ill overweight elderly patients are hypercatabolic and have increased nutrient demands. Nutritional support in these patients is required to provide necessary nutrient substrates and to alter the course and outcome of the disease.
\end{abstract}

Key Words overweight, elderly, critically ill

Nowadays, the global prevalence of overweight and obesity has increased since 1980 to an extent that nearly a third of the world population iscategorized as overweight or obese (1-3). More than 1.4 billion people worldwide were overweight and another 500 million were obese in 2008 according to the World Health Organization. (4). Asia asthe most populous continent is being the new epicenter of the obesity epidemic. The growth of BMI in Asia is above the global average. The increase in BMI was $0.6 \mathrm{~kg} / \mathrm{m}^{2} /$ decade amongst AsiaPacific males and $1 \mathrm{~kg} / \mathrm{m}^{2} /$ decade amongst Southeast Asian females. In terms of percentage of body fat, even with similar body mass index (BMI), obesity in Asians deviates from those of European origin. This diversity made the World Health Organization (WHO) recommended different BMI cut-offs for the Asian population. Higher visceral fat content is linked with increased insulin resistance and predisposes the Asian population to a

E-mail: christina_rusli@yahoo.com distinctly higher degree of cardiovascular and diabetes risk (3).

Obesity has adversely affected almost all physiological functions of the body. It holds a significant public health threat. by increasing the risk of developing multiple disease conditions, such as diabetes mellitus, cardiovascular disease, chronic kidney disease, several types of cancers, an array of musculoskeletal disorders, and poor mental health. All of which have negative effects on the quality of life, work productivity, and healthcare costs for the patients and the government $(3,5)$. Less attention has been paid to this connection, although there is and indication that the constant growth in chronic kidney disease (CKD) prevalence may be closely associated with increasing body weight in obese. Obesity induces cardiovascular and renal diseases through numerous mechanisms, such as hypertension, hyperglycemia, dyslipidemia, inflammation, and atherosclerosis. These ailments often coincide, especially when there is excess visceral fat. This condition has often been referred to as 
"metabolic syndrome" (3-5). The combination of the escalating prevalence of obesity in the general population and the high burden of comorbidities has resulted in an increased number of obese patients requiring admission to the intensive care unit. This condition also caused by the association of obesity with several important pathophysiological derangements of the respiratory, cardiac, and metabolic functions which might generate a further multiorgan impairment when these subjects are exposed to stresses of acute illness (6).

This case report revealed the effect of nutrition therapy support in critically ill overweight elderly patient with heart failure, myocardial infarction, pneumonia, and chronic kidney disease who was admitted in cardiac intensive unit at Wahidin Sudirohusodo hospital, Makassar, Indonesia. Nutritional therapy provided necessary nutrient substrates and altered the course and outcome of the disease.

\section{MATERIALS AND METHODS}

In-patient diagnosis and treatment. In December 2019 , an 80-y old male patient with a history of productive cough and dyspnea for three days was admitted to the cardiac intensive care unit of Wahidin Sudirohusodo Hospital, Makassar, Indonesia. At the time of admission, his vital signs were: blood pressure 110/70 $\mathrm{mm} \mathrm{Hg}$ (on dobutamine $5 \mathrm{mcg} / \mathrm{kg}$ body weight/minute), heart beat 120-150 beats per minute, body temperature $36.5^{\circ} \mathrm{C}$, and respiratory rate 28 times per minute. After medical evaluation, the patient was diagnosed with cardiogenic shock, non-ST segment elevation myocardial infarction (NSTEMI), severe atrial stenosis, severe mitral stenosis, Community-Acquired Pneumonia (CAP), mild hyperkalemia, normalization of ventricular rhythm (NVR) Atrial Fibrillation, and Acute Kidney Injury (AKI) DDx. acute on Chronic Kidney Disease (CKD).

He had a medical history of hypertension and heart disease for the past six years with regular drug consumption (digoxin, furosemide, and simarc). The patient also had prostate surgery fifteen years ago. The patient was a retiree. He was a passive-smokers with no alcohol concumption habit. After one day of admission to the hospital, the patient was consulted to the clinical nutrition department. On the initial assessment we found decrease of oral intake due to shortness of breath, productive cough, and loss of appetite. There was no history of weight loss. Urination was $200 \mathrm{~mL} / 12 \mathrm{~h}$, via catheter while excrement was no discharge for three days.

Nutrition assessment. Anthropometric Measurements. Knee height was $50 \mathrm{~cm}$. Estimated body height was calculated based on knee height measurement and the result was $162 \mathrm{~cm}$. Ideal body weight was $55.8 \mathrm{~kg}$. Mid arm circumference was $30 \mathrm{~cm}$ and estimated body weight was $63.6 \mathrm{~kg}$. The clinical examination results of base crackles and wheezing, irregular heart rhythm, swelling (edema) of feet, and muscle wasting.

Biochemical data, medical test, and procedures. At the initial laboratory examination, we found mild hyper- kalemia, leukocytosis, moderate immune system depletion, decreased of kidney function, mild hypoalbuminemia, dyslipidemia, and hyperuricemia. Biochemical data are described in Table 1. Echocardiogram showed ejection fraction $51 \%$. Result from whole abdomen ultrasonography were first grade fatty liver, bilateral PNC, and cystitis.

Dietary histrory. Typical intake and usual amounts of food (before admitted to the hospital) was that the patient eat regularly, three times a day in the form of white rice two portions for each meal with varied side dishes and vegetables. The patient often ate fruit. The patient like to consume salty foods, coconut-milk based food, and fried foods. The patient did not eat for two days when he was first admitted to the hospital, but after one day of admission, the patient was able to eat five tablespoons of porridge and had some milk. The dietary history data was collected using 24-h recall. Its results exhibited inadequate dietary intake of macronutrients with energy $287.5 \mathrm{kcal}(18 \%)$, protein $9 \mathrm{~g}$ (12\%), carbohydrate $51 \mathrm{~g}(70 \%)$, and fat $7 \mathrm{~g}(21 \%)$.

Nutrition diagnosis

1. Moderate protein energy malnutrition based on Subjective Global Assessment score B proved with inadequate energy intake related to reduced appetite and shortness of breath evidenced by dietary history (287.5 kcal/d).

2. Dyslipidemia, hyperkalemia and hyperuricemia due to chronic kidney disease.

3. Leucocytosis, immune system depletion, and hypoalbuminemia due to hypercatabolic state.

4. Excessive carbohydrate and protein intake related to lack of knowledge regarding nutrition for chronic kidney disease.

Estimated requirements. The estimated energy requirements was $20-25 \mathrm{kcal} / \mathrm{kg}$ body weight/d $( \pm 1,300-$ $1,600 \mathrm{kcal} / \mathrm{d}$ ) when patient was in cardiac intensive care unit with protein $1.2 \mathrm{~g} / \mathrm{kg} / \mathrm{d}$ and $1,900 \mathrm{kcal} / \mathrm{d}$ (calculated using Harris-Benedict equation) with protein $0.6 \mathrm{~g} / \mathrm{kg}$ body weight $/ \mathrm{d}$ when the patient was transferred to general ward.

Nutrition interventions

Medical nutritional therapy objectives:

1. Improve nutritional status with adequate intake of energy, protein, and other nutrients to meet nutrient needs according to critically ill and CKD treatment.

2. Provide nutritional support appropriate to the patient's condition for moderate treatment of energy protein malnutrition, hyperkalemia, leukocytosis, immune system depletion, impaired renal function, hypoalbuminemia, dyslipidemia, and hyperuricemia.

3. Provide macronutrient and micronutrient supplementation that is suitable for the patient's medical condition.

4. Increase patient and his families' awareness regarding the importance of improvement of food choices for helping the treatment process and prevention of morbidity and mortality.

5. Conduct regular monitoring and evaluation to determine the subsequent therapy plan. 


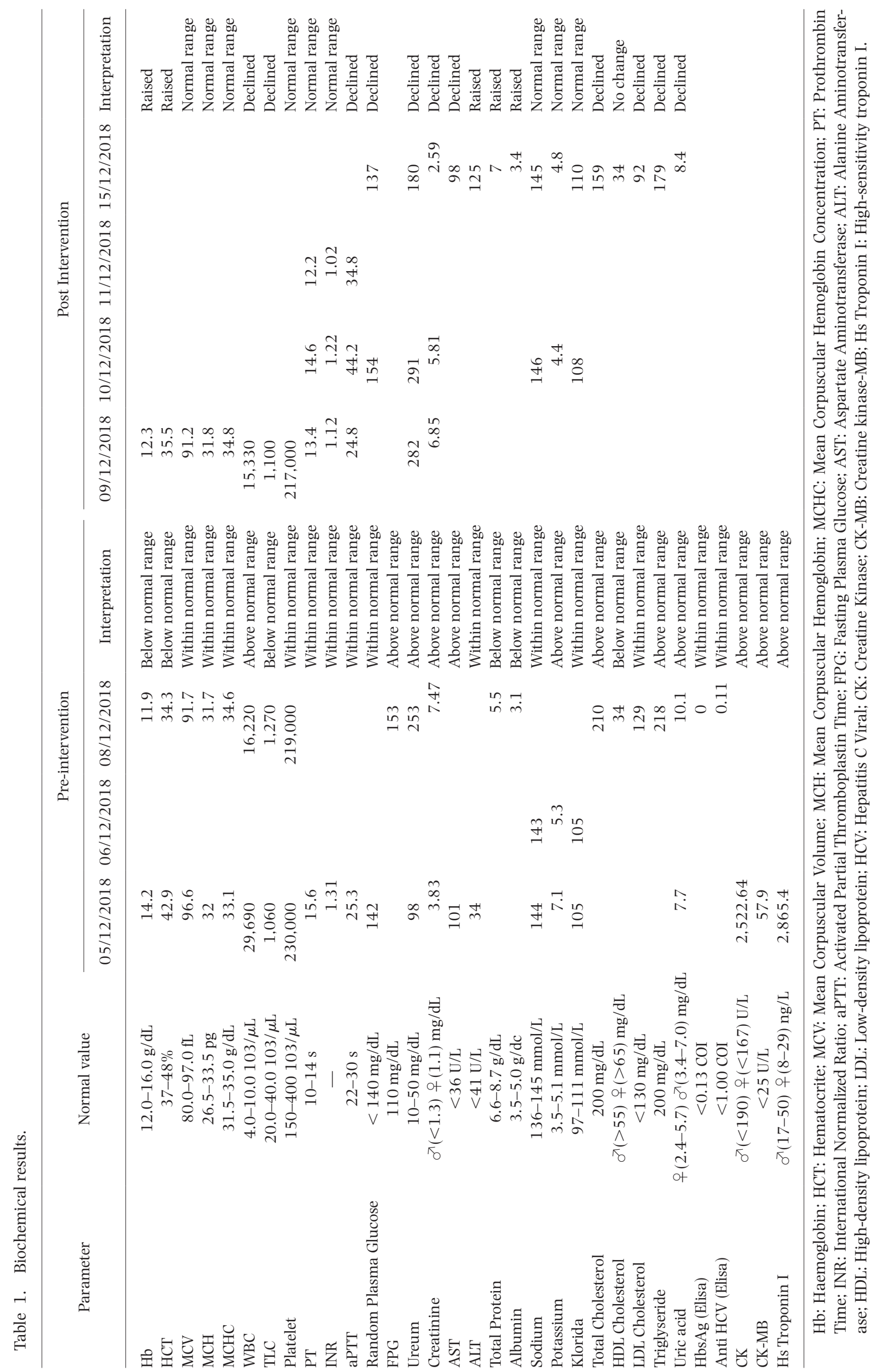


ENERGY (Kcal)

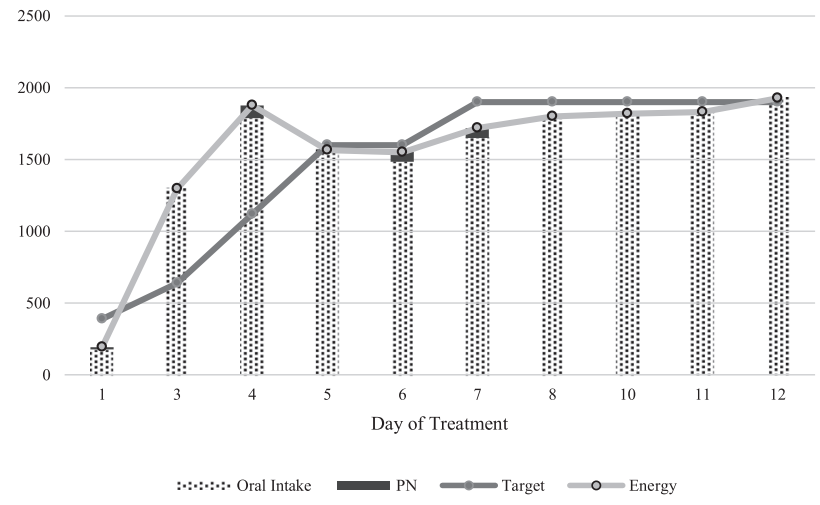

Fig. 1. Daily Intake.

\section{PROTEIN INTAKE (g)}

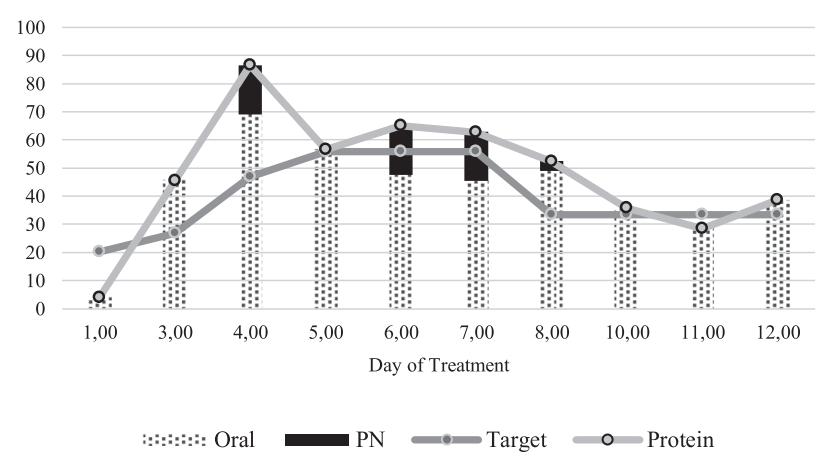

Fig. 2. Protein Intake.

\section{Nutrition prescription}

Nutrition plan. Macronutrients: 45-50\% carbohydrates, 7-16.7\% protein, and 36-38.3\% lipids were recommended. Fluid: No restriction of fluids was instituted.

Food and/or nutrient delivery. The patient was given parenteral nutrition by the internist, and he was asked to eat adequate protein intake (obtained from daily food and formula that were recommended), as well as eating six meals/d. The patient was asked to eliminate high potassium and uric acid foods. The patient was told to continue with nutrient supplements (vitamin B complex, vitamin $C$, curcumin, snakehead fish extract capsules, and zinc).

Nutrition education. The importance of medical nutrition therapy for chronic kidney disease condition was explained, as well as risks and complications associated with his disease. Nutrition education about high and low potassium and uric acid foods was provided, and intake of low potassium and uric acid foods was encouraged by suggesting affordable and preferable food options. High biological value (HBV) protein food sources were suggested to have high protein utilization. Food sources with high potassium and content were discussed. Nutrition plan with different choices from each food group was also explained. An example of a 1-d menu with the recommended food groups and serving sizes was provided to the patient.
CARBOHYDRATE INTAKE (g)

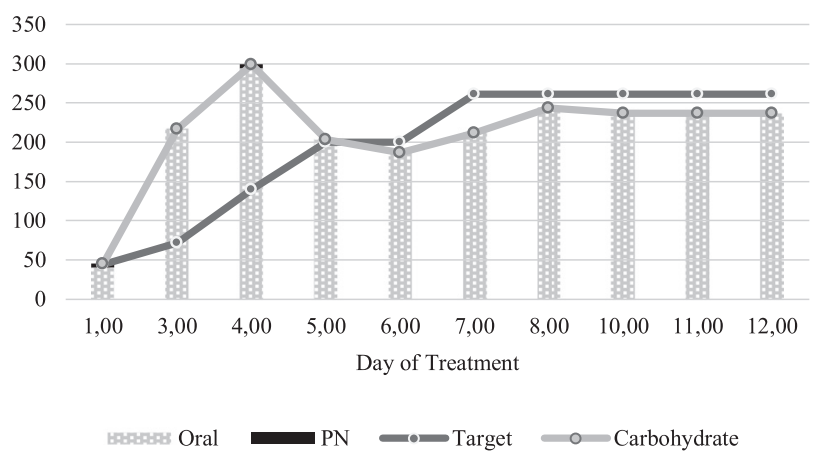

Fig. 3. Carbohydrate Intake.

FAT INTAKE $(\mathrm{g})$

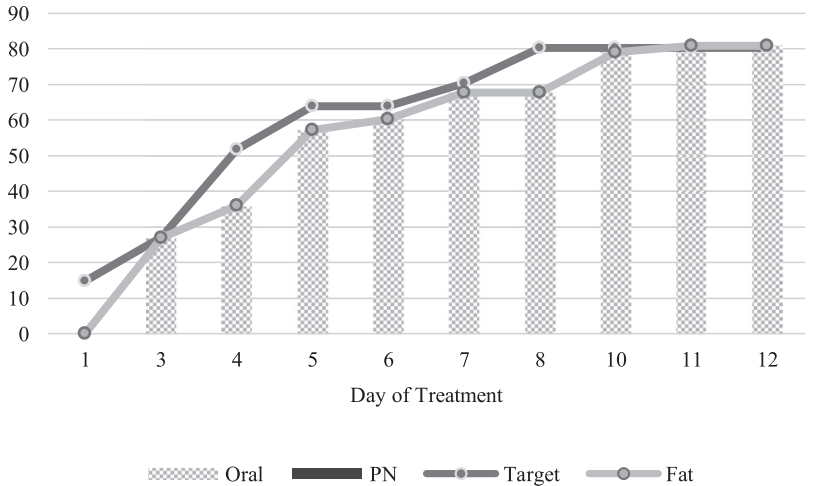

Fig. 4. Fat Intake.

Nutrition assessment and monitoring. In order to keep an eye over the compliance of implementation of nutrition intervention plan, continuous nutrition monitoring was done on daily basis. The patient was monitored during his complete hospitalization period i.e. $13 \mathrm{~d}$. The vital signs charts, laboratory reports, fluid balance sheet, records of clinical signs and 24-h food recall were examined daily.

\section{RESULTS}

The comparison of pre-interventional and post-interventional results of nutrition assessment of the patient revealed that nutrition interventions brought out a remarkable difference in all parameters. Dietary data is also switched towards the recommended intake. Primary positive nutrition changes during hospital stay included adjustment of macronutrients intake according to patient's requirement and reduce intake of high potassium and uric acid foods. At the end of hospital stay, he reported good adherence to the nutrition plan (Figs. 2-4).

In anthropometric measurements, an increase of 0.3 centimeters mid upper arm circumference (from $30 \mathrm{~cm}$ to $30.3 \mathrm{~cm}$ ) was found in the patient. This result was in line with nutritional therapy's goal to maintain patient body weight (Fig. 5). Physical examination for clinical nutrition-specific signs and symptoms also showed 
improvement. Fluid intake was maintained at 1,600 to $1,900 \mathrm{~mL} / \mathrm{d}$. No physical evidence of overhydration was observed.

In biochemical analysis, the lab values for albumin showed a raised trend while lab values for BUN, creatinine, potassium and uric acid showed a declined trend. White blood cell, total lymphocyte count, and lipid profile examination were improved (Table 1). At the end of patient treatment in the hospital, the patient and his family had a better understanding about his illness and nutrition teraphy according to his illness.

\section{DISCUSSION}

One of the main challenges of medical nutrition therapy for chronic kidney disease was meeting the recommendation of energy and protein intake. Nutritional requirements in these patients vary depending on the clinical condition and results of the patient's laboratory examination. This challenge becomes increasingly difficult when patients must be in intensive care. This case report represents one example of nutrition therapy in critically ill patients with chronic kidney disease.

Despite its complexity, the provision of adequate nutrition has shifted as an integral component of supportive ICU care. Up until now, there is still an ongoing debate within the literature regarding the optimal route of delivery, the target dose, and the macronutrient components (proportion of protein and non-protein calories) of nutritional support for critically ill patients. For overweight critical ill patient, the recommended energy intake at acute phase was $20 \mathrm{kcal} / \mathrm{kg}$ body weight and increased in later phase to $25 \mathrm{kcal} / \mathrm{kg}$ body weight. As for protein intake, the recommendation range was $1.2-$ $1.5 \mathrm{~g} / \mathrm{kg}$ body weight/d $(6,7)$.

This recommendation was used when the patient was in the cardiac intensive unit. During the critical illness period, we commonly observed an extensive loss of the patient's total body protein mass. The breakdown of skeletal muscles is the primary source of amino acids for the synthesis of acute-phase proteins and immunoglobulins. Loss of muscle protein happens immediately in critically ill patients and may result in a loss of up to $18 \%$ of muscle mass in the first $10 \mathrm{~d}$ of intensive care unit (ICU) stay. This condition will lead to a marked negative total body nitrogen balance in the patient $(6,8)$. When the patient was admitted to the cardiac intensive case unit, our nutritional target was to fulfill the nutritional requirements and prevent negative nitrogen balance. When an obese patient becomes critically ill, the ICU team is confronted with extra challenges, which puts the obese patient at risk for poor outcomes. On the contrary, the current literature suggests that obese patients have improved ICU outcomes. This phenomenon was called "obesity paradox" in critical setting. Therefore, eventhough our patient is in the overweight category, we did not aim to reduced his body weight (9).

Inflammation plays a significant role in the acute thrombotic events pathophysiology. It is associated with Acute Myocardial Infarction (AMI), which often marked with peripheral leukocytosis and relative neutrophilia.
In acute coronary syndromes, elevated counts, especially neutrophil count, has been correlated with a greater risk of acute ischemic events and with adverse angiographic findings. Studies have verified that white blood cells (WBC) represents a marker of inflammation, and the amount of inflammation may be directly associated with plaque instability and LV dysfunction, which results in recurrent short and long term cardiac events (10).

A clinical syndrome characterized by an inability to restore homeostasis between pro-inflammatory and anti-inflammatory systems and the persistence of a severe anti-inflammatory state is called immunoparalysis. Lymphocytes depletion or lymphocytopenia is prevalent among ICU patients. A study reported that $75 \%$ of ICU patients have moderate to severe lymphocytopenia $(11,12)$. Adequate delivery of nutrients is fundamental for sustaining metabolism and body composition among critically ill patients. Nutritional deficiencies can point to immune system dysfunction. $(12,13)$ Nutritional therapy to improve the immune system and reduce the incidence of malnutrition is carried out by meeting the patient's energy and protein requirements. We also gave antioxidants and micronutrients supplementation that enhance the immune system in the form of vitamin $C$, zinc, and snakehead fish extract capsules. We gave curcumin as an anti-inflammatory agent to reduce the inflammatory process so that it can also improve the immune system.

Hypoalbuminemia is a well known condition in elderly patients, particularly in hospitalized patients, and in times of malnutrition, chronic illness, and critical ilness. The pathophysiology of hypoalbuminemia includes a decrease in amino acids or energy supply, diminished liver synthesis, increased loss (renal or gastro-intestinal), enhanced tissue catabolism or distributional issues $(14,15)$. To improve hypoalbuminemia in this patient, nutritional therapy was given in the form of adequate intake of energy and protein by oral and parenteral, and supplementation of snakehead fish extract capsules. Snakehead fish is an alternative source of albumin due to its compounds of high protein, fat, water, and minerals (especially zinc). Zinc acts as an antioxidant that protects cells, stimulates the process of wound healing, regulates expression in lymphocytes and proteins, improves appetite and stabilizes body weight (16).

Obesity and hyperlipidemia are the most prevalent independent risk factors of chronic kidney disease (CKD). This suggesting that lipid accumulation in the renal parenchyma is deleterious to the renal function. Of all kinf of fat, the non-esterified fatty acids (also known as free fatty acids, FFA) are particularly detrimental to the kidneys (17). The patient was on stage 5 renal failure (GFR $6.2 \mathrm{~mL} / \mathrm{min}$ ) when he was first admitted on the hospital. When the patient was transferred to general ward, the nutritional therapy was switched according to recommendation for CKD patients. A neutral or slightly positive nitrogen balance can be maintained with a low quantity $(\sim 0.6 \mathrm{~g} / \mathrm{kg} / \mathrm{d})$ 
but high quality (HBV) protein diet and adequate energy intake (30-35 kcal/kg/d) for non-dialysis CKD patients. Protein-energy wasting (PEW) indicates a concomitant loss of protein and energy stores in patients with kidney dysfunction. This condition tends to develop and advance with CKD progression. When CKD and ESRD patients have a suboptimal energy supply, their body start to catabolize muscle to provide needed energy, leading to protein malnutrition $(18,19)$. In chronic kidney disease patients, the goal of nutritional therapy was to prevent the occurrence of proteinenergy malnutrition. In this patient, adequate nutrition was provided so that renal function increases without any weight loss. These improvements marked by increased upper arm circumference as one of the anthropometric monitoring and increased in renal function (GFR $22.4 \mathrm{~mL} / \mathrm{min}$ ).

High triglycerides, low HDL levels, and normal or slightly reduced cholesterol-LDL are characteristics that usually found in CKD patients. These characteristics were responsible for cardiovascular events (20-22). CKD nutritional management often includes recommendations to reduce protein intake and to regulate electrolyte imbalances. However, little attention has been paid to lipid consumption, probably due to conflicting data emerging from several trials about their role in CKD progression. Nevertheless, quantitative and quality dietary fat intake should be monitored, considering that dyslipidemia represents an independent risk factor for cardiovascular disease (20).

Olive oil is one of the functional food that has high mono-unsaturated fatty acids content and also other minor, biologically active, components, such as vitamins, minerals, and polyphenols. These dietary polyphenols have been reported to act as an antioxidant and anti-inflammatory agent that improve endothelial dysfunction and lipid profile (23). We suggest olive oil to this patient to meet his energy requirements and to replace saturated fats consumption to keep his blood cholesterol within normal levels and protect blood lipids from oxidative stress. When the patient was discharged from the hospital, there was an improvement in his lipid profile.

Kidneys play a fundamental role in sustaining total body potassium stores. When confronted with fluctuations in potassium intake and shifts in transcellular electrolytes, the body's capability to maintain normal potassium level (normokalemia) is increasingly compromised in the present of progressive CKD. Hyperkalemia, as one of complication of CKD, has direct effects on cardiac conduction that might contribute to cardiovascular mortality in the patients (24). Gout is a also a disease that associated with a progressive functional impairment, reduced quality of life, lost productivity and increased mortality. In recent observational studies, they confirmed that both hyperuricaemia and gout are possible risk factors for progression of chronic kidney disease (CKD). This emerging evidence suggesting that the treatment and control of gout are especially important among patients with impaired kidney function, which would lead to measurable clinical benefits. (25). In this case report, the condition of hyperkalemia and hyperuricemia that we found in the patient was due to existing kidney function disorders. This patient was advised to undergo hemodialysis therapy to manage the ailment, but he refused it. Therefore, treatment of hyperkalemia and hyperuricemia in this patient was supported by restricting the intake of foods that contain high potassium or uric acid levels. We also educated the patient and his families about the type of foods that high in potassium or uric acid levels and food processing methods so that potassium intake can be maintained. Also, restrictions of dietary protein intake was carried out to prevent further deterioration of kidney function so that the patient can maintain stabilization of the condition of normokalemia in his body.

Nutritional therapy performs a significant role in chronic kidney disease patient's treatment. Nutrition education, as one part of nutrition therapy, must include patients and their families to guarantee the accomplishment of therapeutic targets. The purpose of nutrition education in patients with chronic kidney disease is to provide awareness of the significance of nutritional therapy in preventing patient morbidity and mortality, as well as the amount and type of food intake that patients must consume when the patient was being hospitalized or when the patient was discharged from the hospital (26).

Hence, the Nutrition Care Process is successfully applied to the respective case study, and the core job of Medical Nutrition Therapy is also fulfilled accurately. This case study can be used as an example to follow for the up-coming clinicians and researchers in the field of medical nutrition therapy.

\section{CONCLUSION}

This is a case that report the challenge of applying the nutrition care process for overweight patient with CKD during critical ill. Obesity paradox has a unique role within the health care team responsible for treating these patients in critical ill setting. Clinical nutritionist must provide specific renal nutrition therapy interventions and nutrition education and counseling.

\section{Disclosure of state of COI}

No conflicts of interest to be declared.

\section{REFERENCES}

1) Chung Y, Ding C, Magkos F. 2019. The epidemiology of obesity. Metabolism 92: 6-10.

2) Han SJ, Boyko EJ. 2018. The evidence for an obesity paradox in Type 2 diabetes mellitus. Diabetes Metab J 42(3): 179-187.

3) Mukhopadhyay A. et al. 2018. Higher BMI is associated with reduced mortality but longer hospital stays following ICU discharge in critically ill Asian patients. Clin Nutr ESPEN 1-6. doi: 10.1016/j.clnesp.2018.08.009

4) Hall ME, Silva AA, Juncos LA, Wang Z, Hall JE. 2014. Obesity, hypertension, and chronic kidney disease. Int J Nephrol Renovasc 4: 75-88.

5) Gadde KM, Martin CK, Berthoud H-R, Heymsfield SB. 
2018. Obesity pathophysiology and management. JACC 71: 69-84.

6) Diet and Nutrition in Critical Care. 2015. Springer Science + Business Media.

7) Singer P, et al. 2019. Very high intact-protein formula successfully provides protein intake according to nutritional recommendations in overweight critically ill patients: a double-blind randomized trial. Clin Nutr $\mathbf{3 8}$ : 48-79.

8) Zanten ARH Van, et al. 2018. Very high intact-protein formula successfully provides protein intake according to nutritional recommendations in overweight critically ill patients: a double-blind randomized trial. Crit Care 22: $1-12$.

9) Patel JJ, et al. The Critical Care Obesity Paradox and implications for nutrition Support. Curr Gastroenterol Rep 18: 1-8.

10) Jan AF, Habib S, Naseeb K, Khatri MA, Zaman KS. 2011. High total leukocyte count and heart failure after myocardial infarction. PAKISTAN Hear J 44: 8-17.

11) Chang CH, Byun CG, Lee MW, Jeong JY, Shin CS. 2003. The value of the total lymphocyte count as a risk index of hospital infection in critically ill patients. Korean $J$ Anesthesiol 44: 1-4.

12) Manzoli TF, et al. 2016. Lymphocyte count as a sign of immunoparalysis and its correlation with nutritional status in pediatric intensive care patients with sepsis: A pilot study. Clinics 71: 644-649.

13) Kosałka K, Wachowska E, Słotwinski R. 2017. Disorders of nutritional status in sepsis-facts and myths. Gastroenterol Rev 12: 73-82.

14) Moujaess E, et al. 2017. The Therapeutic use of human albumin in cancer patients' management. Crit Rev Oncol Hematol 120: 203-209.

15) Moustafa AA, Halawany AS Al, Rafa MF. 2018. Evaluation of hypoalbuminemia as a predictor of clinical outcome in critically ill children in Alexandria University Children's Hospital. J Med Sci Clin Res 6: 32299-32306.

16) Restiana Taslim NA, Bukhari A. 2010. Pengaruh Pem- berian Ekstrak Ikan Gabus Terhadap Kadar Albumin dan Status Gizi Penderita HIV/AIDS yang Mendapatkan Terapi ARV. e-jurnal Repos. Univ. Hasanuddin.

17) Gai Z, et al. 2019. Lipid accumulation and chronic kidney disease. Nutrients 11: 1-21.

18) Zha Y, Qian Q. 2017. Protein nutrition and malnutrition in CKD and ESRD. Nutrients 9: 1-19.

19) Piccoli GB, et al. 2016. Patient survival and costs on moderately restricted low-protein diets in advanced CKD: Equivalent survival at lower costs? Nutrients 8: 1-11.

20) Visconti L, et al. 2016. Lipid disorders in patients with renal failure: Role in cardiovascular events and progression of chronic kidney disease. J Clin Transl Endocrinol 6: 8-14.

21) Mihalache A, Garneata L, Mircescu G. 2018. Nutritional patterns in chronic kidney disease. Arch Balk Med Union 53: 427-433.

22) Wanner C, Tonelli M, Kidney Disease: Improving Global Outcomes Lipid Guideline Development Work Group Members. 2014. KDIGO Clinical Practice Guideline for Lipid Management in CKD: summary of recommendation statements and clinical approach to the patient. Kidney Int 85: 1303-1309.

23) Tsartsou E, Proutsos N, Castanas E, Kampa M. 2019. Network meta-analysis of metabolic effects of olive-oil in humans shows the importance of olive oil consumption with moderate polyphenol levels as part of the Mediterranean diet. Front Nutr 6: 1-11.

24) Tomey MI, Winston JA. 2014. Cardiovascular pathophysiology in chronic kidney disease: Opportunities to transition from disease to health. Ann Glob Heal 80: $69-76$.

25) Mohammed E, et al. 2019. Prevalence and treatment of gout among patients with chronic kidney disease in the Irish health system: A national study. PLoS One 14: $1-14$.

26) Taslim NA, et al. 2015. Standar Pelayanan Gizi klinik. (Departemen Ilmu Gizi FK-UNHAS-Masagena Press). 